\title{
Abnormal Vital Capacity
}

National Cancer Institute

\section{Source}

National Cancer Institute. Abnormal Vital Capacity. NCI Thesaurus. Code C78715.

A pulmonary function test result indicating an abnormal vital capacity as compared to the predicted value. 